\title{
Electronic structure calculations for substitutional copper and monovacancies in silicon
}

\author{
C D Latham ${ }^{1,3,4}$, M Ganchenkova ${ }^{1}, \mathbf{R}$ M Nieminen ${ }^{1}$, S Nicolaysen ${ }^{2}$, M Alatalo ${ }^{3}$, \\ S Öberg ${ }^{4}$, and P R Briddon ${ }^{5}$ \\ ${ }^{1}$ Laboratory of Physics, Helsinki University of Technology, PO Box 1100, FIN-02015 HUT, Finland \\ ${ }^{2}$ Department of Physics, University of Oslo, PO Box 1048 Blindern, N-0316 Oslo, Norway, and \\ Institute for Microsystem Technology, PO Box 2243, N-3103 Tonsberg, Norway \\ ${ }^{3}$ Department of Electrical Engineering, Lappeenranta University of Technology, PO Box 20, \\ FIN-53851 Lappeenranta, Finland \\ ${ }^{4}$ Department of Mathematics, Luleå University of Technology, SE-97187 Luleå, Sweden \\ ${ }^{5}$ Physics Centre, School of Natural Science, University of Newcastle upon Tyne, Newcastle, \\ NE1 7RU, UK
}

Emailcdl@fyslab.hut.fi

Received 21 August 2005

Accepted for publication 24 November 2005

Published 17 August 2006

Online at stacks.iop.org/PhysScr/T126/61

\begin{abstract}
Two different computer program packages based on the self-consistent local-spin-density approximation-AIMPRO and VASP-are employed in this study of substitutional copper $\mathrm{Cu}_{\mathrm{Si}}$ and monovacancies $V_{\mathrm{Si}}$ in silicon, including the effects of their charge state. The programs differ in the types of basis sets and pseudopotentials they use, each with their own relative merits, while being similar in overall quality. This approach aims to reduce uncertainty in the results, particularly for small or subtle effects, where the risk is greatest that the conclusions are affected by artifacts specific to a particular implementation. The electronic structures of the two defects are closely related, hence they are expected to behave in a similar manner. For both defects structural distortions resulting in lower point group symmetries than $T_{d}$ (the highest possible) are found. This is in good agreement with the results of previous studies of $V_{\mathrm{Si}}$. Much less is known about symmetry-lowering effects for $\mathrm{Cu}_{\mathrm{Si}}$; however, the electronic levels of $\mathrm{Cu}_{\mathrm{Si}}$ have been measured accurately, while those for $V_{\mathrm{Si}}$ are less accessible. Calculating them is a challenging task for theory. The strategy we adopt, based purely on comparing total energies of supercells in different charge states, with and without model defects, reproduces the three known levels for $\mathrm{Cu}_{\mathrm{Si}}$ reasonably well. Satisfactory results are also obtained for $V_{\mathrm{Si}}$, so far as can be judged for this more complex case.
\end{abstract}

PACS numbers: 61.72.Bb, 61.72.Ji, 71.15.Mb

\section{Introduction}

The silicon monovacancy $V_{\mathrm{Si}}$ and substitutional transition metal atoms $T M_{\mathrm{Si}}$ with full or nearly full $d$-shells posses closely related electronic structures. They are described well in terms of one-electron models developed by Watkins [1,2]. As a consequence of the Jahn-Teller effect, structural distortions occur that lower the symmetry of the defects from the $T_{d}$ point group. In an undistorted configuration, with $T_{d}$ symmetry, $V_{\mathrm{Si}}$ and $T M_{\mathrm{Si}}$ have a degenerate triplet $t_{2}$ state in the forbidden band. Therefore, depending on how this is affected by structural relaxation, they may have up to six possible charge states. For $T M_{\mathrm{Si}}$ there is also an interaction between the $t_{2}$ state in the bandgap and the $d$-electrons which lie in the valence band.

In the case of $V_{\mathrm{Si}}$, five charge states $(+2,+1,0,-1,-2)$ and their corresponding four levels apparently exist. However, the Jahn-Teller effect is sufficiently strong in the neutral state to reverse the energetic ordering of the $(0 /+1)$ and $(+1 /+2)$ transitions $[3,4]$. In other words, it has 'negative- $U$ ' and $V_{\mathrm{Si}}^{+1}$ 
is energetically unstable. Only three levels are observed for substitutional copper $\mathrm{Cu}_{\mathrm{Si}}$, corresponding to charge states +1 , $0,-1$, and -2 [5-10]. There is no experimental information about the defect's symmetry at present.

The Jahn-Teller distortions for $V_{\mathrm{Si}}$ and $T M_{\mathrm{Si}}$ posses two components. The main one is tetragonal in character: alone, it gives the defects $D_{2 d}$ symmetry. The tetragonal distortion may occur in two opposite, senses. This determines the relative energetic ordering of the resulting electronic states [11]. We will label these two senses $A$ and $B$. They correspond to different relative lengths for the six $\mathrm{Si}$-Si distances surrounding the centre. For the $D_{2 d}$ point group, four of them belong to one set of equivalent $\mathrm{Si}-\mathrm{Si}$ lengths, and two belong to a second set of equivalent lengths. Type- $A$ distortion has the pair longer than the fourfold set; type- $B$ is the other way around. Type$A$ distortion splits the $t_{2}$ state into a singlet $a_{1}$ state above a doublet $e$ state, while type- $B$ does the opposite. $T M_{\mathrm{Si}}$ defects relax in the $A$-direction [12]. Silicon monovacancies relax in the opposite sense, $B$ [1].

When the system contains sufficient electrons to occupy the $e$ state, a weaker trigonal distortion is expected: it lowers the symmetry to $C_{2 v}$. The twofold equivalent Si-Si lengths in the $D_{2 d}$ case become unequal. This splits the $e$ state into orbitals of $b_{1}$ and $b_{2}$ character. Spin-orbit coupling may also affect the splitting of these states for $T M_{\mathrm{Si}}$ defects [12].

\section{Methods}

The underlying theory used here is the local-density approximation (LDA) or local-spin-density approximation (LSDA) of density functional theory (DFT), depending on the net electronic spin. Existing theoretical methods appear to perform poorly at estimating the energies of electrical levels in semiconductors. The sources of errors responsible for this may be divided into three main parts: the underlying theory, the approximations required to construct each specific implementation, and the model used to represent the particular system of interest. In this study we compare the results of two different computer program packages based on the same underlying theory, applied to $V_{\mathrm{Si}}$, and $\mathrm{Cu}_{\mathrm{Si}}$. By doing this we aim to reduce the uncertainties in the results, and possibly identify particular strengths or weaknesses of each program package. Moreover, being less constrained by finite computer resources than in the past, we can also make a better assessment of how well electrical levels may be calculated.

To conduct our study, we employ the AIMPRo [13-15] and vASP [16-19] program packages. The model defects are constructed in supercells based on $3 \times 3 \times 3$ conventional cubic unit cells in the diamond structure stacked together (216 atoms). The volume of the supercells is held fixed at the size corresponding to the equilibrium lattice parameter $a$ calculated by the respective methods, while the atoms are moved to minimise their total energy and forces on them. This aims to simulate more nearly an infinite crystal where $a$ would not change in response to the presence of a single defect. During the energy minimisation procedure, the atomic coordinates may optionally be constrained to a particular point group symmetry, or allowed to relax freely.

Within the AIMPRO formalism, the core electrons of atoms
Table 1: Calculated and measured values for the lattice parameter $a$ and bulk modulus $B_{0}$ of $\mathrm{Si}$.

\begin{tabular}{llc}
\hline method & $a(\AA)$ & $B_{0}(\mathrm{GPa})$ \\
\hline AIMPRO & 5.3948 & 97.0 \\
VASP + VUS & 5.390 & 87.7 \\
VASP + PAW & 5.403 & 72.8 \\
measured & 5.431021 & 97.9 \\
\hline
\end{tabular}

are represented by pseudopotentials constructed according to the norm-conserving Hartwigsen-Goedecker-Hutter (HGH) scheme [20]. The exchange-correlation energy contribution is evaluated according the formula described by Perdew and Wang [21]. The vasP package includes pseudopotentials based on the Vanderbilt ultrasoft construction [22, 23] (VUS), and the projector augmented wave method [24, 25] (PAW). The $\mathrm{Cu}-3 d$ electrons are included explicitly with the HGH and PAW schemes, while they are not for the VUS pseudopotential. In both AIMPRO and VASP, the bandstructure is sampled according to the Monkhorst-Pack (MP) scheme [26]. The convergence criterion for total energies is $\epsilon \lesssim 10^{-4} \mathrm{eV}$. To achieve this requires MP $-2^{3} \mathbf{k}$ points. These are folded according to the symmetry of the system, and shifted to avoid the $\Gamma$ point. Both packages represent the charge density using a plane-wave basis. The kinetic energy cutoff needed to meet the convergence criterion depends on the types of atoms present, and the pseudopotentials used. Trial calculations are performed to determine the appropriate settings. The wavefunction is also described by a plane-wave basis in the vasP package, while AIMPRO uses a Gaussian basis. To obtain satisfactory results it is necessary to have four exponents per atom. Suitable prefactors provide orbital symmetries with maximum angular momentum $l_{\max }=2$ for all four on $\mathrm{Cu}$ atoms, while on $\mathrm{Si}$ atoms the two largest exponents have $l_{\max .}=1$ and the two smallest have $l_{\max .}=2$.

Electrical levels are calculated from the change in total energy when electrons are added to or removed from the system. When supercells are charged, a compensating background charge of equal and opposite magnitude is added to prevent the total energy from becoming divergent. However, the potential is undefined. There are several methods to obtain a reference. The one that we use is to compare the change in energy when electrons are added or removed, with that for a pure, ideal silicon supercell of equivalent size. The potential of the top of the valence band $E_{v}$ is determined by calculating the difference in energy between a neutral, perfect supercell and one with charge $q=+1$, while the bottom of the conduction band $E_{c}$ is equal to the electron affinity of a perfect supercell. The bandgap $E_{g}=E_{c}-E_{v}$ depends on the size of the supercell: the greater its volume $V$, the smaller the gap, converging ultimately at the LDA value. The main factor responsible for this effect is simply that the electron or hole concentration varies as $1 / V$. We may also calculate the energies of defects with respect to one another. This is the so-called 'marker method'. Full details of the procedures for calculating electrical levels are given in Ref. [27]. 
Table 2: Calculated and measured electrical levels for $V_{\mathrm{Si}}$ and $\mathrm{Cu}_{\mathrm{Si}}$. The AIMPRo results include the use of different symmetry constraints. For $V_{\mathrm{Si}}$, where two point groups are given, the first is the symmetry in positive and neutral states, and the second is the symmetry in negative charge states. The vasp calculations do not have any symmetry constraints.

\begin{tabular}{|c|c|c|c|c|c|c|c|}
\hline \begin{tabular}{l|l}
$d$ & $\backslash$
\end{tabular}$q^{\prime}$ & $0 /+2$ & $+1 /+2$ & $0 /+1$ & $-1 / 0$ & $-2 /-1$ & $-2 / 0$ & method \\
\hline$\overline{V_{\mathrm{Si}}\left(T_{d}\right)}$ & & $E_{v}+0.07$ & $E_{v}+0.18$ & $E_{c}-0.34$ & $E_{c}-0.24$ & & AIMPRO \\
\hline$V_{\mathrm{Si}}\left(D_{2 d} / C_{3 v}\right)$ & $E_{v}+0.00$ & $E_{v}+0.05$ & $E_{v}-0.04$ & $E_{c}-0.31$ & $E_{c}-0.43$ & $E_{c}-0.37$ & AIMPRO \\
\hline$V_{\mathrm{Si}}\left(D_{2 d} / C_{2 v}\right)$ & $E_{v}+0.00$ & $E_{v}+0.05$ & $E_{v}-0.04$ & $E_{c}-0.29$ & $E_{c}-0.24$ & & AIMPRO \\
\hline$V_{\mathrm{Si}}$ & $E_{v}+0.09$ & $E_{v}+0.13$ & $E_{v}+0.05$ & exists & exists & & Ref. [1] \\
\hline $\mathrm{Cu}_{\mathrm{Si}}\left(D_{2 d}\right)$ & & & $E_{v}+0.17$ & $E_{c}-0.50$ & $E_{c}-0.24$ & & AIMPRO \\
\hline $\mathrm{Cu}_{\mathrm{Si}}\left(T_{d}\right)$ & & & $E_{v}+0.21$ & $E_{c}-0.40$ & $E_{c}-0.35$ & & AIMPRO \\
\hline $\mathrm{Cu}_{\mathrm{Si}}\left(\approx D_{2 d}\right)$ & & & $E_{v}+0.07$ & $E_{c}-0.45$ & $E_{c}-0.18$ & & VASP + VUS \\
\hline $\mathrm{Cu}_{\mathrm{Si}}\left(\approx T_{d}\right)$ & & & $E_{v}+0.17$ & $E_{c}-0.34$ & $E_{c}-0.26$ & & VASP + PAW \\
\hline $\mathrm{Cu}_{\mathrm{Si}}$ & & & $E_{v}+0.21$ & $E_{c}-0.69$ & $E_{c}-0.17$ & & Refs. [8-10] \\
\hline
\end{tabular}

\section{Results and discussion}

This report represents work in progress, and as such some of the results should be regarded as preliminary. This applies especially to the results obtained with the vasp code, where some technical issues remain unresolved at present. In particular, structural optimisation of both defects has proved problematic for VASP, possibly due to them having fairly flat energy surfaces in the region of their minima. At present we are not yet confident that our results obtained with the vasP code for $V_{\mathrm{Si}}$ are sufficiently reliable to report in full.

Both methods are able to reproduce the lattice parameter $a$ and bulk modulus $B_{0}$ of $\mathrm{Si}$ well. The results, compared with the measured values are shown in Table 1 . Note that $B_{0}$ calculated by the AIMPRo method uses the Birch equation of state, while the VASP calculations use the harmonic approximation. If anharmonic terms are taken into account, then $B_{0}$ is expected to be $\sim 5 \%$ larger than without them.

The bandgap $E_{g}$ represents the least well defined, and least accurate quantity that we calculate. This is a well-known deficiency of the underlying formalism, however, it will be seen that using our strategy for calculating electrical levels means it does not lead to any further difficulties. Both AIMPRo and vASP give similar results: $E_{g}$ (AIMPRO) $\approx 0.71 \mathrm{eV}$, while $E_{g}($ VASP $) \approx 0.60 \mathrm{eV}$. The vasP result does not depend on whether the pseudopotential type is VUS or PAW.

All of the defect levels calculated here are sufficiently deep that the energies of donors can be given with respect to $E_{v}$ and acceptors with respect to $E_{c}$. This is the preferred situation. Then the sign of the charged ideal supercell used to calculate the reference potential is the same as the defect supercell, hopefully giving better cancellation of systematic errors. None are so shallow that their energy is between the theoretical and true bandgap. If this were the case, then it would be necessary to use the opposite ideal-crystal band for the reference potential. The nearest case to this occurring is the midgap level of $\mathrm{Cu}_{\mathrm{Si}}$. Calculated and measured electrical levels for both defects are presented in Table 2. The results obtained with the AIMPRO package include different symmetry constraints to show their effect.

While both program packages are set to use similar thresholds in terms of energies and forces for deciding when structural optimisation is complete, the outcome shows some differences. When no symmetry constraints are applied, both methods typically have residual, random differences for equivalent lengths between atoms, related by symmetry, that are $\lesssim 0.01 \AA$. The differences between inequivalent lengths for the six Si-Si distances surrounding the central $\mathrm{Cu}$ atom or the vacancy are typically $\sim 0.1 \AA$, making it quite apparent what the symmetry is.

It is found with the AIMPRo package that the direction of the main tetragonal distortion, giving $D_{2 d}$ symmetry, is in the expected direction for each defect: type- $B$ for $V_{\mathrm{Si}}$, and type- $A$ for $\mathrm{Cu}_{\mathrm{Si}}$. The vasp package also finds that $V_{\mathrm{Si}}^{0}$ exhibits a type- $B$ $D_{2 d}$ distortion (results for other charge states are inconclusive at present). VASP yields approximately a type- $A D_{2 d}$ distortion for $\mathrm{Cu}_{\mathrm{Si}}$ when the VUS pseudopotential is used, but there is no significant distortion from $T_{d}$ symmetry with the PAW method. To demonstrate the effect of constraining the symmetry of the defect to be $T_{d}$, the levels calculated using AIMPRo for this configuration are also given in Table 2.

Notice in Table 2 that the donor levels calculated by AIMPRO for $V_{\mathrm{Si}}$ exhibit negative- $U$ (giving reversed order) when $T_{d}$ symmetry is permitted to break. Both donor transitions are also slightly too deep, so the calculated $(0 /+1)$ level lies below the top of the valence band.

The calculated donor transition for $\mathrm{Cu}_{\mathrm{Si}}$ is closer to the measured value than for $V_{\mathrm{Si}}$. Both AIMPRo and vASP-PAW yield equal energies for the $(0 /+1)$ level of $\mathrm{Cu}_{\mathrm{Si}}$, in spite of having different structures for the defect.

Regardless of constraint choice, no clear evidence is seen for the expected trigonal component of the distortion for $\mathrm{Cu}_{\mathrm{Si}}$. A possible explanation for this discrepancy may be found in the way that the bandstructure is sampled: the degeneracy of the electronic system is lifted without a structural distortion. The lack of spin-orbit coupling in the theory, and finite size of the model are two other factors that may also be affecting the outcome.

The situation is more complicated for $V_{\mathrm{Si}}$. While the optimised structure in positive and neutral charge states calculated by AIMPRo has $D_{2 d}$ symmetry as expected, it must be constrained to be $C_{2 v}$ in the negative charge states, otherwise the lowest energy structure is a $C_{3 v}$ symmetry split-vacancy configuration with nearly, but not exactly $D_{3 d}$ symmetry. The outcomes in both cases are shown in Table 2. Previously, Puska 
et al. found that $V_{\mathrm{Si}}$ in negative charge states adopts a splitvacancy configuration as well [28]. They provide a review of earlier work; see also Ref. [29].

According to our calculations, the energy differences between the $C_{2 v}$ and $C_{3 v}$ configurations of $V_{\mathrm{Si}}$ in the -1 and -2 charge states are $0.02 \mathrm{eV}$ and $0.20 \mathrm{eV}$, respectively. Thus, the high stability of the $C_{3 v}$ configuration in the -2 charge state means that $V_{\mathrm{Si}}$ also has negative- $U$ for the acceptor levels, according to this model. Probably, but not certainly, this is incorrect, and the model constrained to have $C_{2 v}$ symmetry in negative charge state is the true situation. If the splitvacancy configuration does exist, then according to the JahnTeller theorem it should have lower symmetry than $D_{3 d}$ in the -1 charge state, while it may be $D_{3 d}$ symmetry in the -2 state. Little is known from experiment at present about the negative charge states of $V_{\mathrm{Si}}$ other than they apparently exist.

The calculated energy for the $(-1 / 0)$ acceptor level of $\mathrm{Cu}_{\mathrm{Si}}$ near midgap is somewhat deeper than the measured value, while the second acceptor is shallower. The Jahn-Teller effect moves these levels apart, making the $(-1 / 0)$ level shallower and the $(-2 /-1)$ level deeper, each by $\sim 0.1 \mathrm{eV}$. Thus, our calculations appear to underestimate the size of the JahnTeller effect, and especially for the vAsP-PAW calculation, where no significant distortion from $T_{d}$ symmetry occurs. It is reasonable to suppose that a similar pattern will be found for the acceptor states of $V_{\mathrm{Si}}$ if they are measured, and the expected $C_{2 v}$ model without negative- $U$ prevails.

If the measured electrical levels for $\mathrm{Cu}_{\mathrm{Si}}$ are compared with the three corresponding electrical levels of $V_{\mathrm{Si}}$ instead of using ideal, pure supercells, and we use the $C_{2 v}$ model in negative charge states, then the first acceptor level is about $0.2 \mathrm{eV}$ shallower, while the donor and double acceptor change much less. The three energies calculated with AIMPRO are: $E(0 /+1) \approx E_{v}-0.01 \mathrm{eV}, E(-1 / 0) \approx E_{c}-0.48 \mathrm{eV}$, and $E(-2 /-1) \approx E_{c}-0.17 \mathrm{eV}$.

\section{Conclusions}

The structures and electrical levels for $V_{\mathrm{Si}}$ and $\mathrm{Cu}_{\mathrm{Si}}$ calculated using the AIMPRO method are mostly in good agreement with accepted models and experimental observations. The main exception is the negatively charged states of $V_{\mathrm{Si}}$, where the calculations appear to disagree with the conventional model. The vasP program package is also able to reproduce reasonably well the three electrical levels of $\mathrm{Cu}_{\mathrm{Si}}$, although calculations based on the PAW scheme apparently underestimate the size of the Jahn-Teller effect. A more satisfactory result appears to be obtained using the VUS pseudopotential, in spite of neglecting the $\mathrm{Cu}-3 d$ electrons.

Overall, these results give grounds for believing that electrical levels of defects in semiconductors-which are considered to be difficult to estimate accurately-can be calculated reliably using conventional DFT-based methods, provided it is done with appropriate care.

\section{Acknowledgements}

This research has been supported by Lappeenranta University of Technology, and the Academy of Finland through its Centers of Excellence Programme. Computer resources have been provided by the National Supercomputer Centre in Sweden (NSC), the Centre for Scientific Computing (CSC) in Finland, and Computer Services for Academic Research (CSAR) in the United Kingdom. SÖ and CDL also thank the Swedish Research Council, Vetenskapsrådet (VR) for providing financial support.

\section{References}

[1] Watkins G D 1992 Deep centers in semiconductors 2nd edn, ed S T Pantelides (Yverdon: Gordon and Breach Science) ch III, p 177

[2] Watkins 1983 G D Physica B 117-118 9

[3] Baraff G A, Kane E O and Schlüter M 1979 Phys. Rev. Lett. 43956

[4] Baraff G A, Kane E O and Schlüter M 1980 Phys. Rev. B 215662

[5] Lemke K 1986 Phys. Status Solidi A 95665

[6] Brotherton S D, Ayres J R, Gill A, van Kesteren H W and Greidanus F J A M 1987 J. Appl. Phys. 621826

[7] Istratov A A, Hieslmair H, Flink C, Heiser T and Weber E R 1997 Appl. Phys. Lett. 712349

[8] Knack S 2002 PhD thesis Technischen Universität Dresden

[9] Knack S, Weber J and Lemke H 1999 Physica B 273-274 387

[10] Knack S, Weber J, Lemke H and Riemann H 2002 Phys. Rev. B 65165203

[11] Anderson F G, Ham, F S and Watkins G D 1992 Phys. Rev. B 453287

[12] Watkins G D and Williams P M 1995 Phys. Rev. B 5216575

[13] Jones R and Briddon P R 1998 Identification of defects in semiconductors vol 51A ed M Stavola (Boston: Academic) ch VI

[14] Briddon P R and Jones R 2000 Phys. Status Solidi B 217131

[15] Coutinho J, Jones R, Briddon P R and Öberg S. 2000 Phys. Rev. B 6210824

[16] Kresse G and Hafner J 1993 Phys. Rev. B 47558

[17] Kresse G 1993 PhD thesis Technischen Universität Wien

[18] Kresse G and Furthmüller J 1996 Comp. Mater. Sci. 615

[19] Kresse G and Furthmüller J 1996 Phys. Rev. B 5411169

[20] Hartwigsen C, Goedecker S and Hutter J 1998 Phys. Rev. B, 583641

[21] Perdew J P and Wang Y 1992 Phys. Rev. B 4513244

[22] Vanderbilt D 1990 Phys. Rev. B 417892

[23] Laasonen K, Pasquarello A, Car R, Lee C and Vanderbilt D 1990 Phys. Rev. B 4710142

[24] Blöchl P E 1994 Phys. Rev. B 5017953

[25] Kresse G and Joubert D 1999 Phys. Rev. B 591758

[26] Monkhorst H J and Pack J D 1976 Phys. Rev. B 135188

[27] Latham C D et al. 2005 Phys. Rev. B 72235205

[28] Puska M J, Pöykkö S, Pesola M and Nieminen R M 1998 Phys. Rev. B 581318

[29] Probert M I J and Payne M C 2003 Phys. Rev. B 67075204 